\title{
Role of Radiative-Convective Feedbacks in Spontaneous Tropical Cyclogenesis in Idealized Numerical Simulations $\mathscr{0}$
}

\author{
Allison A. Wing And SuZana J. CAmargo \\ Lamont-Doherty Earth Observatory, Columbia University, Palisades, New York \\ ADAM H. SOBEL \\ Department of Earth and Environmental Sciences, and Department of Applied Physics and Applied Mathematics, \\ Columbia University, New York, New York
}

(Manuscript received 21 December 2015, in final form 28 April 2016)

\begin{abstract}
The authors perform 3D cloud-resolving simulations of radiative-convective equilibrium (RCE) in a rotating framework, with interactive radiation and surface fluxes and fixed sea surface temperature. A tropical cyclone is allowed to develop spontaneously from a homogeneous environment, rather than initializing the circulation with a weak vortex or moist bubble (as is often done in numerical simulations of tropical cyclones). The resulting tropical cyclogenesis is compared to the self-aggregation of convection that occurs in nonrotating RCE simulations. The feedbacks leading to cyclogenesis are quantified using a variance budget equation for the column-integrated frozen moist static energy. In the initial development of a broad circulation, feedbacks involving longwave radiation and surface enthalpy fluxes dominate, which is similar to the initial phase of nonrotating self-aggregation. Mechanism denial experiments are also performed to determine the extent to which the radiative feedbacks that are essential to nonrotating self-aggregation are important for tropical cyclogenesis. Results show that radiative feedbacks aid cyclogenesis but are not strictly necessary.
\end{abstract}

\section{Introduction}

Self-aggregation is a mode of convective organization found in radiative-convective equilibrium (RCE) simulations, in which there is a spontaneous transition from randomly distributed to organized convection despite homogeneous boundary conditions. Previous studies have found that feedbacks involving clouds, water vapor, and radiation are important for permitting selfaggregation (Tompkins 2001; Bretherton et al. 2005; Stephens et al. 2008; Muller and Held 2012; Jeevanjee and Romps 2013; Wing and Emanuel 2014; Muller and

\footnotetext{
Supplemental information related to this paper is available at the Journals Online website: http://dx.doi.org/10.1175/JAS-D-150380.s1.

Corresponding author address: Allison A. Wing, LamontDoherty Earth Observatory, Columbia University, 61 Route 9W, P.O. Box 1000, Palisades, NY 10964.

E-mail: awing@1deo.columbia.edu
}

Bony 2015; Wing and Cronin 2016). Analogous behavior occurs in single-column and cloud-resolving models in weak temperature gradient mode (WTG; Sobel et al. 2007; Sessions et al. 2010). Self-aggregation has primarily been studied in a nonrotating framework, but it has been hypothesized to be important to tropical cyclogenesis (e.g., Bretherton et al. 2005; Nolan et al. 2007).

Despite its importance, there remain significant gaps in our understanding of tropical cyclogenesis. In recent years, the "marsupial pouch" has emerged as a promising theoretical framework. Tropical cyclogenesis tends to occur near the critical layer of a tropical wave (Dunkerton et al. 2009), in which there is a pouch that is favorable for the development of a tropical cyclone. This "pouch" region is a preferred region for genesis not only because it is protected from the entrainment of dry air from the environment but also because convection in the pouch repeatedly moistens the column. The relationship between precipitation and saturation fraction is also an essential part of the genesis theory presented by 
Raymond et al. (2007). Preservation of a moist area that is favorable for convection is also fundamental to the phenomenon of self-aggregation, suggesting a link between aggregation and tropical cyclogenesis.

Previous studies of RCE in a rotating framework have examined the sensitivity of tropical cyclogenesis and tropical cyclone intensity, size, and frequency to environmental parameters and horizontal resolution (Nolan et al. 2007; Held and Zhao 2008; Khairoutdinov and Emanuel 2013; Zhou et al. 2014; Boos et al. 2016; Reed and Chavas 2015). Nolan et al. (2007) suggested that the initial development of a broad circulation in a case of spontaneous tropical cyclogenesis was due to the same radiative-convective feedbacks that cause nonrotating self-aggregation-a hypothesis we examine further. Several recent studies have examined the effects of interactive radiation on tropical cyclones, including modulation of the structure by cloud radiative forcing (Bu et al. 2014), the sensitivity of genesis to the presence of a diurnal cycle (Melhauser and Zhang 2014), and the importance of explicit radiation (as opposed to idealized Newtonian cooling) for achieving a statistically steady axisymmetric hurricane (Hakim 2011). These studies suggest that radiation can influence tropical cyclones, but with the exception of Davis (2015), who noted that radiation that interacts with clouds and water vapor aids the development of coherent rotating structures in idealized simulations, the impact of radiative feedbacks on tropical cyclogenesis remains relatively unexplored.

In this study, we investigate tropical cyclogenesis in the context of self-aggregation and test the hypothesis that both are instigated by the same radiative-convective feedbacks. We apply an analysis framework based on a variance budget equation for the column-integrated frozen moist static energy to quantify the physical mechanisms leading to cyclogenesis in rotating RCE simulations. We directly compare cyclogenesis to the development of a self-aggregated cluster in nonrotating RCE simulations.

\section{Simulation design}

We simulate statistical radiative-convective equilibrium (RCE) using version 6.8.2 of the System for Atmospheric Modeling (SAM; Khairoutdinov and Randall 2003) cloudsystem-resolving model. We use the SAM one-moment microphysics parameterization, a Smagorinsky-like scheme for subgrid-scale turbulence, and for shortwave and longwave radiation, the Rapid Radiative Transfer Model (RRTM; Mlawer et al. 1997; Clough et al. 2005; Iacono et al. 2008). The use of these particular parameterizations may affect the details and robustness of selfaggregation [e.g., the sensitivity to radiation scheme noted by Wing and Cronin (2016)], but a systematic exploration of these sensitivities is not pursued here. We employ an $f$ plane, with a Coriolis parameter $f=5 \times 10^{-5} \mathrm{~s}^{-1}$. To facilitate comparison between spontaneous tropical cyclogenesis and nonrotating self-aggregation, we perform an additional simulation that has no rotation.

In general, large domains are needed for self-aggregation to occur (Muller and Held 2012). Recent studies that have performed periodic $f$-plane simulations at cloudresolving resolutions have used domain lengths between 960 and $4000 \mathrm{~km}$ (e.g., Davis 2015; Boos et al. 2016; Schecter 2013, 2011; Khairoutdinov and Emanuel 2013; Nolan et al. 2007). Therefore, we employ a grid of $512 \times$ $512 \times 64$, with $3-\mathrm{km}$ horizontal resolution (a domain length of $1536 \mathrm{~km}$ ) and doubly periodic lateral boundaries. The upper boundary is a rigid lid at $28 \mathrm{~km}$, with a sponge layer extending from 19 to $28 \mathrm{~km}$; the vertical grid has 64 levels and variable spacing, with 8 levels in the lowest kilometer and 500-m spacing above $3 \mathrm{~km}$. The lower boundary is a fixed sea surface temperature of $305 \mathrm{~K}$. The solar insolation is fixed at $413 \mathrm{~W} \mathrm{~m}^{-2}$, approximately the tropical annual mean; there is no diurnal cycle.

Rather than using a weak vortex or moist bubble to initialize the model, we allow a circulation to develop spontaneously from an initially homogeneous environment. The model is initialized from an average sounding from the final 20 days of a 100-day RCE simulation with the same boundary conditions but a much smaller domain $(96 \times 96$ grid points in the horizontal) and with a small amplitude of random thermal noise in the lowest five levels (an amplitude of $0.1 \mathrm{~K}$ in the lowest level, decreasing linearly to $0.02 \mathrm{~K}$ in the fifth level). This initial random noise is varied to generate a five-member ensemble of simulations.

We perform mechanism denial experiments in which the degree of interactive radiation is varied. In the first, "rad_homog," the radiative heating rates are horizontally homogenized at each vertical level and time step before being applied. This removes feedbacks resulting from the spatial variation of longwave and shortwave radiative heating. In the second experiment, "LWrad_qfix," the profile of water vapor mixing ratio that enters the longwave radiation calculation is fixed at a constant profile (constant in both space and time) taken from the domainand time-mean of a simulation on a smaller domain at the same sea surface temperature that does not aggregate. This removes the longwave-water vapor feedback but retains longwave-cloud feedbacks and the shortwave radiation feedback.

\section{Results}

Spontaneous tropical cyclogenesis is found to occur in the rotating RCE simulation at $305 \mathrm{~K}$. The system 
(a) Day 15

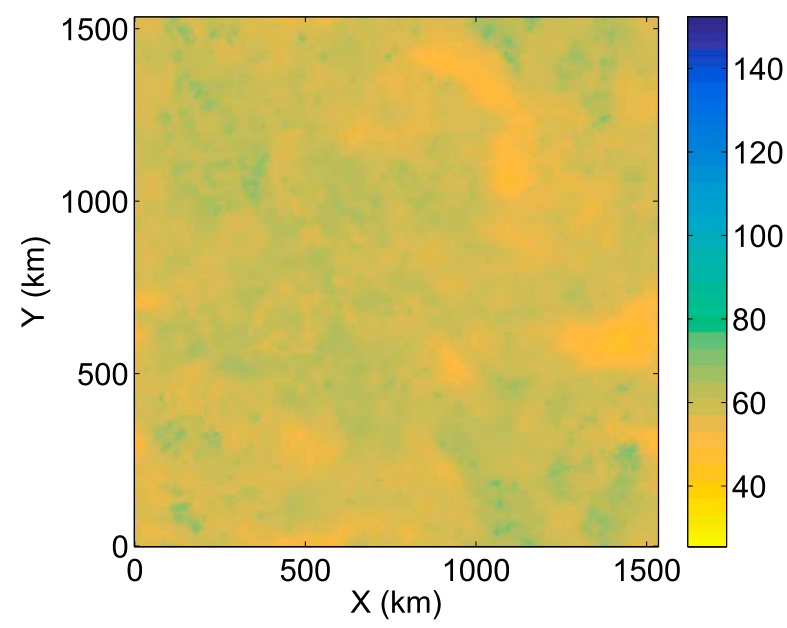

(c) Day 45

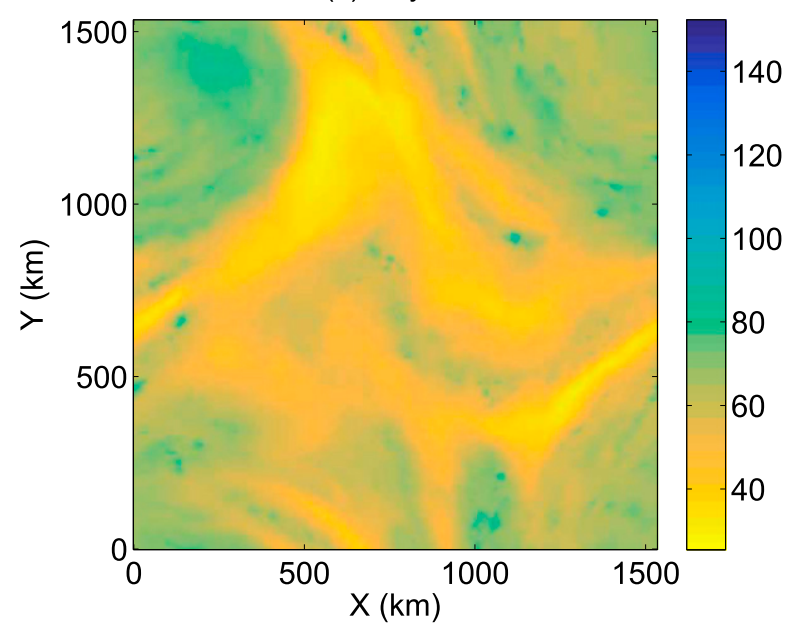

(b) Day 30

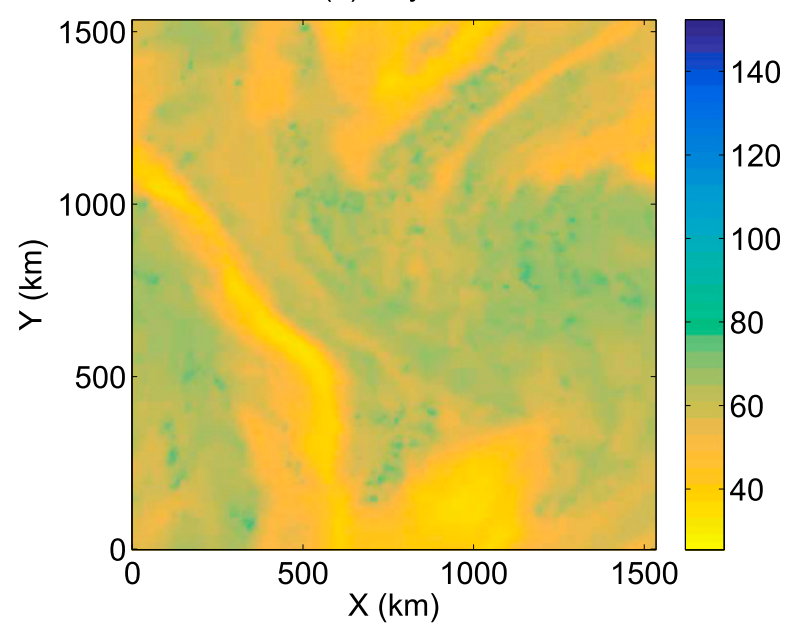

(d) Day 60

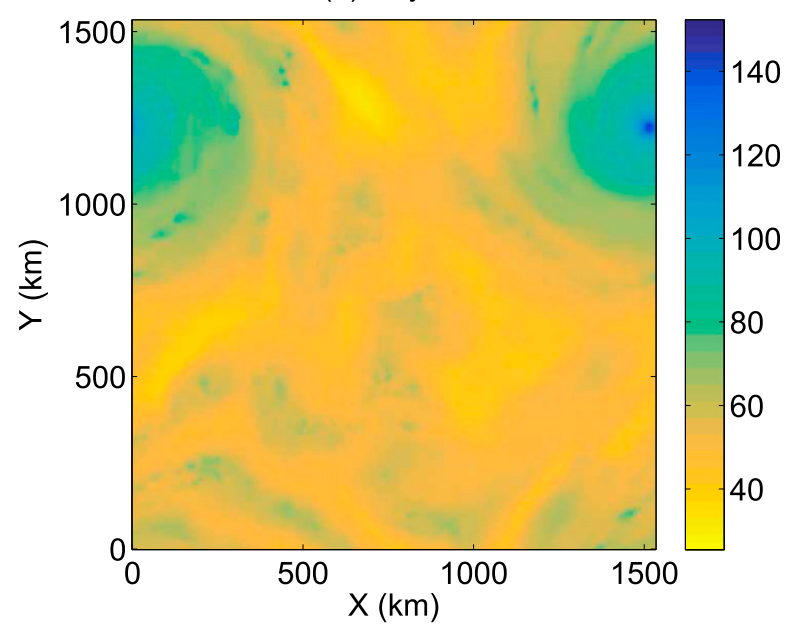

FIG. 1. Hourly mean precipitable water $(\mathrm{mm})$ for simulation at $305 \mathrm{~K}$ with rotation $\left(f=5 \times 10^{-5} \mathrm{~s}^{-1}\right)$.

exhibits qualitatively similar behavior in simulations with sea surface temperatures of 300 and $303 \mathrm{~K}$, in a simulation with a horizontal domain that is 4 times larger in area than the control simulation $(1024 \times 1024$ grid points, yielding a domain length of $3072 \mathrm{~km}$ ), and in a simulation with half the grid spacing $(1.5 \mathrm{~km}$ instead of $3 \mathrm{~km})$. Spontaneous tropical cyclogenesis also occurs in simulations with sea surface temperatures of 285 and $295 \mathrm{~K}$ (not shown), which are below the threshold for nonrotating aggregation found by Wing and Emanuel (2014). However, more recent work (Wing and Cronin 2016) suggests that there may not be such a temperature threshold when larger domains are utilized. The dependence of both nonrotating and rotating aggregation on sea surface temperature thus remains an unsolved problem.

Plan views of hourly average precipitable water $(\mathrm{PW})$ at 15-day increments in the control simulation are shown in Fig. 1. At day 15, the precipitable water is nearly homogenous throughout the domain, indicating convection that is randomly spaced. By day 30 , there are several patches of drier air, and by day 45 , the moister areas have begun rotating. This is in contrast to nonrotating self-aggregation, in which a dry patch expands to force all convection into a single cluster (Wing and Emanuel 2014). This morphological difference [also noted by Davis (2015)] is clear in animations of the evolution of precipitable water in the rotating and nonrotating simulations (see supplemental material). The development of a midtropospheric vortex precedes genesis (not shown), as in Davis (2015), but unlike his simulations, where multiple moist vortices form and then merge, here there is a single large moist area that spins up, which suggests that the precise aggregation and genesis mechanisms could be sensitive to model details. 

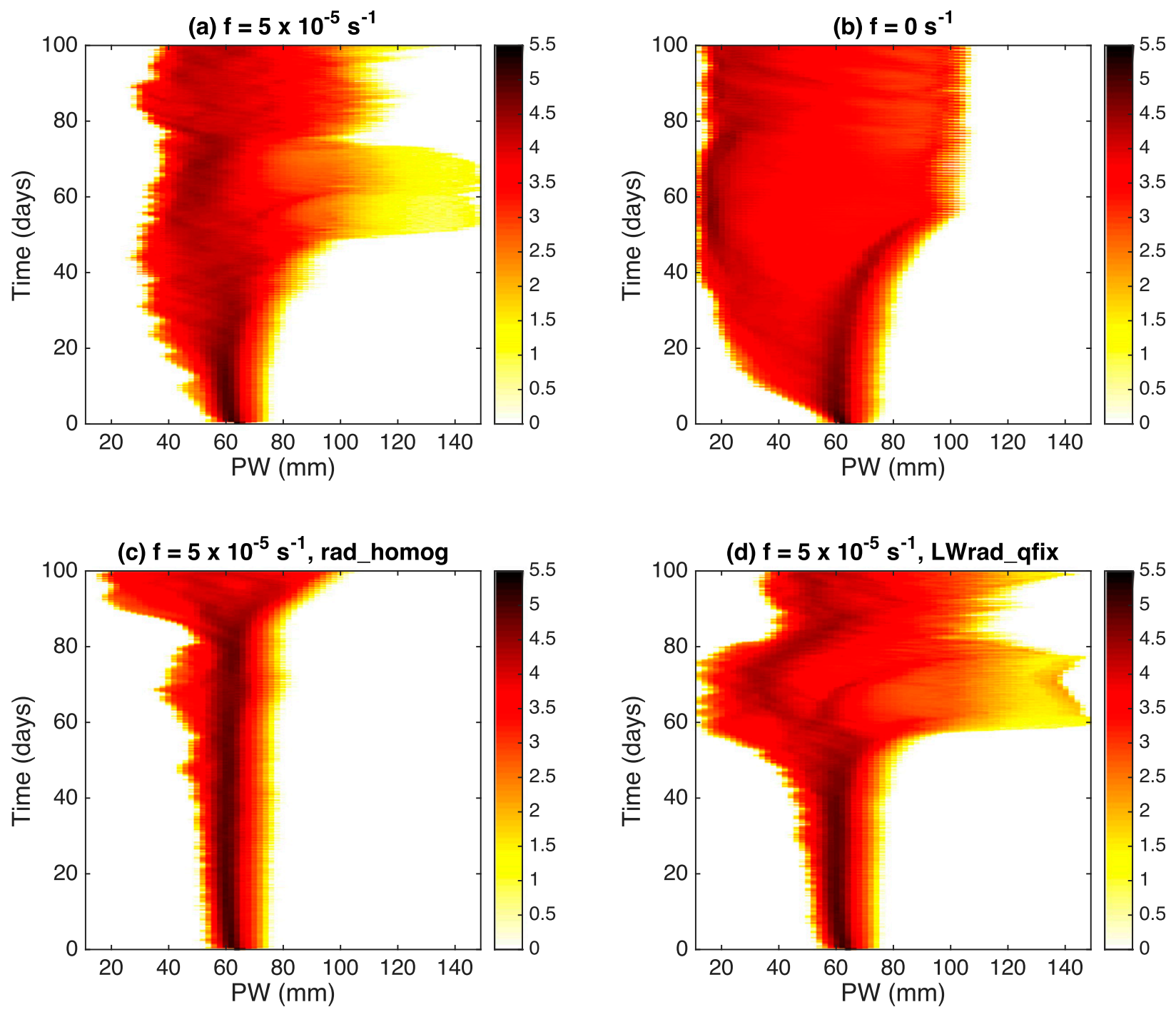

FIG. 2. Histogram of hourly mean precipitable water (2-mm bins) as a function of time for simulations at $305 \mathrm{~K}$ (a) with rotation, (b) without rotation, (c) with rotation but without radiative feedbacks, and (d) with rotation but without longwave-water vapor feedbacks. The logarithm of the counts in each bin is plotted.

We speculate that the difference in evolution between nonrotating and rotating aggregation is related to the strength of the surface enthalpy flux-wind speed feedback in the moist regions. In the early stages of aggregation, there are stronger surface winds in the moist regions in the rotating simulation than in the nonrotating simulation. These stronger surface winds in the rotating simulation, presumably produced by vortex stretching due to surface convergence associated with convection and ascending motion in the moist regions, enhance surface fluxes there and amplify the moist anomalies more than occurs in the nonrotating simulation (not shown). Other factors may also contribute to the suppression of a growing dry patch in the rotating simulation, such as a suppression of radiative feedbacks in dry regions by the outflow of clouds from the developing tropical cyclone or a kinematic straining and destruction of dry patches by the circulation. A detailed analysis of these different factors will be the subject of future work.

By day 60 , the tropical cyclone has intensified and has very high values of precipitable water in the core of the storm (Fig. 1d). These extreme PW values are higher than those found in the nonrotating simulation, as indicated by comparing the evolution of the distribution of PW between the two simulations (Fig. 2). In both simulations, the PW distribution widens, but the driest PW values, near where the peak of the PW distribution is, do not fall as low in the rotating simulation as they do in the nonrotating simulation (Fig. 2). 
(a) Max Wind

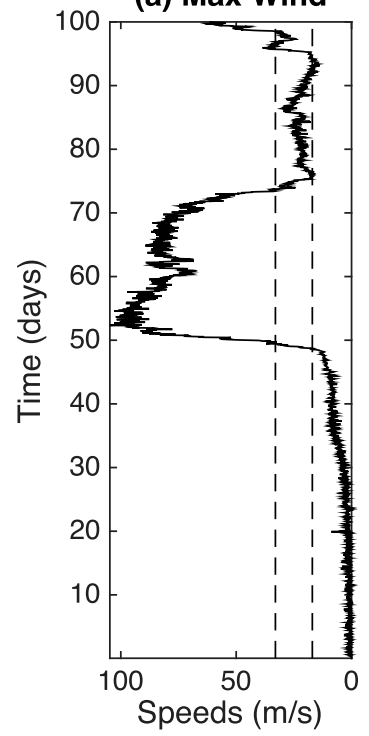

(b) Az. Mean Tangential Wind

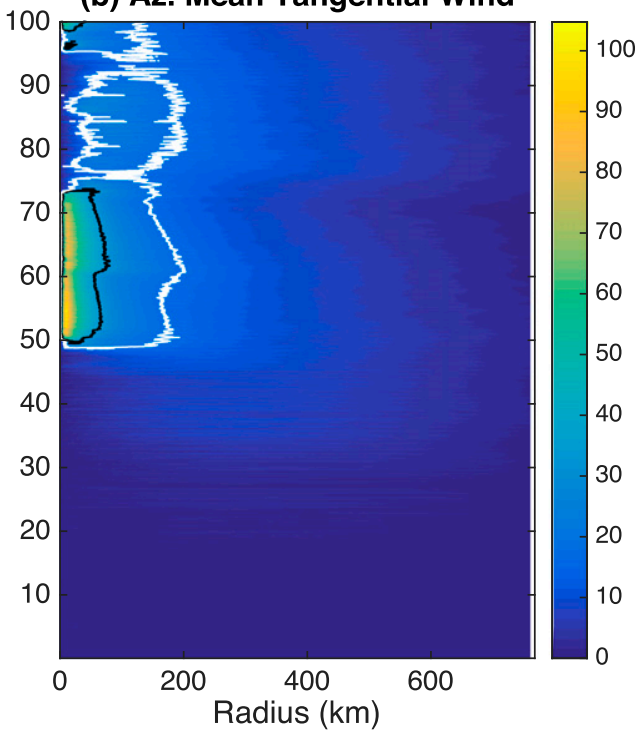

(c) Az. Mean Radial Wind

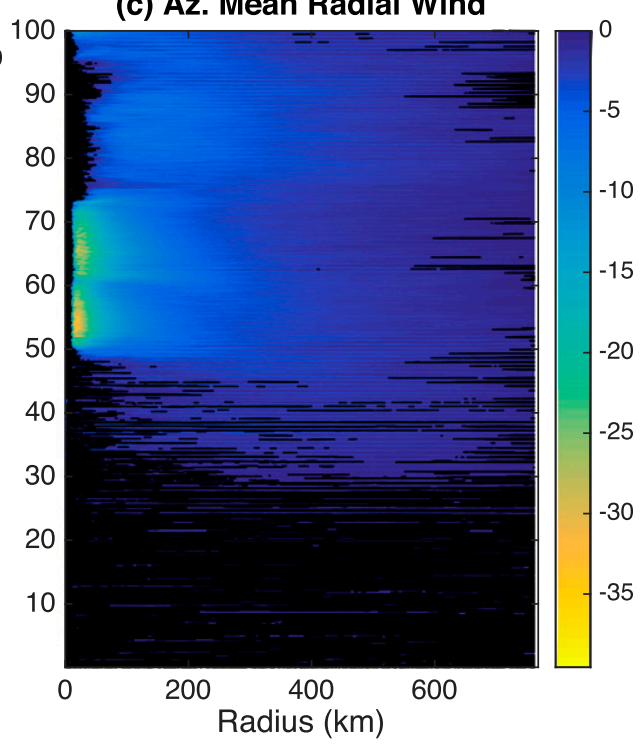

FIG. 3. Evolution of (a) maximum azimuthal-mean tangential wind, (b) azimuthal-mean tangential wind, and (c) azimuthal-mean radial wind (all in m s$\left.~^{-1}\right)$, at $z=194 \mathrm{~m}$, for simulation at $305 \mathrm{~K}$ with rotation $\left(f=5 \times 10^{-5} \mathrm{~s}^{-1}\right)$. Dashed black lines in (a) indicate wind speeds of $17 \mathrm{~m} \mathrm{~s}^{-1}$ (tropical storm strength) and $33 \mathrm{~m} \mathrm{~s}^{-1}$ (hurricane strength). The white and black contours in (b) are contours of 17 and $33 \mathrm{~m} \mathrm{~s}{ }^{-1}$ winds, respectively. In (c), black indicates winds of $0 \mathrm{~m} \mathrm{~s}^{-1}$ or faster. The color bars are saturated at 0 .

In the rotating simulation, the tropical cyclone persists in a quasi-steady state for almost 20 days before rapidly weakening to tropical storm strength, as indicated by a narrowing of the PW distribution as well as the evolution of the tropical cyclone wind field (Fig. 3). To compute the tangential and radial wind components, we found the center of the tropical cyclone according to the location of minimum pressure at the lowest model level $(37 \mathrm{~m})$ and interpolated the horizontal wind to radial coordinates. The evolution of the azimuthal-mean tangential and radial winds emphasizes the spontaneous nature of the tropical cyclogenesis; for the first $\sim 30$ days of the simulation, there is no coherent circulation (and the location of minimum pressure is not truly a "center"). By day 40, a broad circulation has formed, which gradually strengthens and contracts toward smaller radii before rapidly intensifying just prior to day 50 . The circulation remains coherent as the tropical cyclone later weakens and, near the end of the simulation, reintensifies. This type of variability is also exhibited in simulations at other sea surface temperatures and domain sizes.

The time to "genesis," defined here as the first time the maximum wind speed reaches hurricane strength $\left(33 \mathrm{~m} \mathrm{~s}^{-1}\right)$ and continues to increase, is 49 days in the control simulation (Fig. 3a). This metric varies between 26 and 63 days in a five-member ensemble of simulations in which the initial random noise in the model is perturbed (Fig. 4a). There is substantial variability in the strength of the tropical cyclone throughout each of the simulations, and the spread in the time to reach hurricane strength indicates that there is a significant stochastic component to spontaneous tropical cyclogenesis. The time for nonrotating aggregation to occur also varies, by about 15 days, in a similar ensemble of nonrotating RCE simulations (Wing 2014).

\section{Analysis}

The column-integrated frozen moist static energy variance budget (Wing and Emanuel 2014) is applied to quantify the different feedback processes controlling tropical cyclogenesis. This analysis framework has been applied to self-aggregation (Wing and Emanuel 2014; Wing and Cronin 2016; Arnold and Randall 2015) and realistic modeled tropical variability (Bretherton and Khairoutdinov 2015), and a similar approach has been used in theoretical (Sobel and Maloney 2012), observational (Sobel et al. 2014; Yokoi and Sobel 2015), and modeling (Andersen and Kuang 2012; Arnold and Randall 2015) studies of the Madden-Julian oscillation. The frozen moist static energy (referred to herein as $h$ ) is conserved in adiabatic displacements and its column integral is unchanged by convection; it is defined as

$$
h=c_{p} T+g z+L_{v} q-L_{f} q_{c, i}
$$

where $c_{p}$ is the specific heat of dry air at constant pressure, $L_{v}$ is the latent heat of vaporization, $q$ is the water vapor mixing ratio, $L_{f}$ is the latent heat of fusion, and $q_{c, i}$ 
(a) Max Azimuthal Mean Tangential Wind
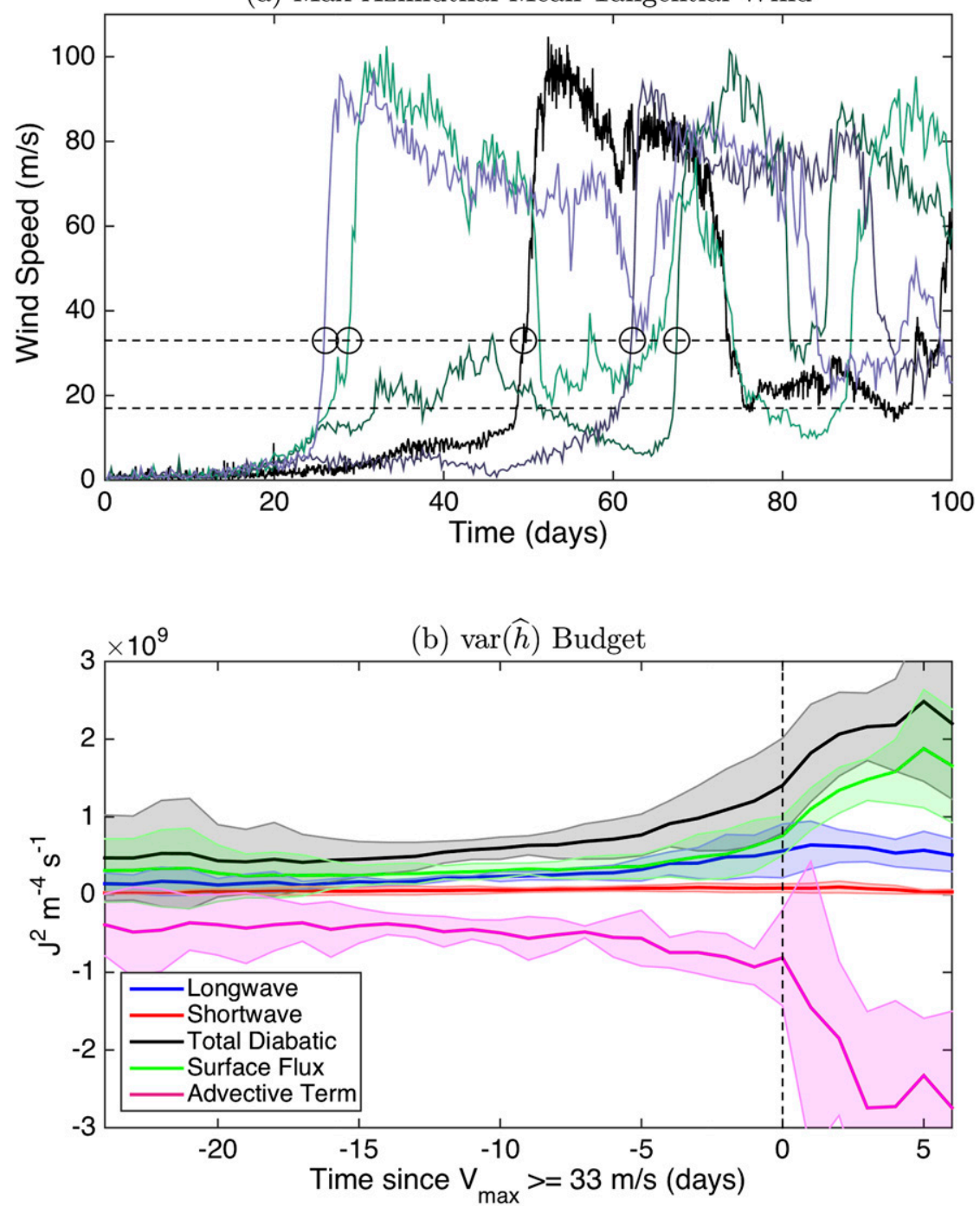

FIG. 4. Five-member ensemble of simulations at $305 \mathrm{~K}$ with rotation $\left(f=5 \times 10^{-5} \mathrm{~s}^{-1}\right)$. (a) The evolution of the maximum azimuthal-mean tangential wind for each ensemble member, where the control simulation (Fig. 3) is in black. The dashed lines indicate wind speeds of $17 \mathrm{~m} \mathrm{~s}^{-1}$ (tropical storm strength) and $33 \mathrm{~m} \mathrm{~s}^{-1}$ (hurricane strength). The circles indicate the first time the wind speed reaches hurricane strength and continues to increase, defined as genesis. (b) The contributions to the growth rate of $\operatorname{var}(\hat{h})$ for the ensemble members shown in (a), where the solid line is the ensemble mean and the shading indicates the standard deviation. The ensemble members are aligned at the times indicated by the circles in (a), which is defined as time 0 on the time axis in (b). The ensemble mean and standard deviation are shown only for times prior to genesis in which all ensemble members have data, up until the time of maximum wind speed.

is the condensed ice water mixing ratio. The variability of column frozen moist static energy is closely tied to that of precipitable water. As shown in Fig. 2, as the simulation evolves to an aggregated state with a tropical cyclone, the distribution of precipitable water widens considerably. This is consistent with a dramatic increase in the spatial variance of column-integrated frozen moist static energy, motivating the use of a budget equation to quantify the different processes contributing to this increase in variance. The budget equation for the evolution of column-integrated frozen moist static energy variance $\left\langle{\widehat{h^{\prime}}}^{2}\right\rangle \equiv \operatorname{var}(\hat{h})$, the spatial mean of the squared anomaly of $\hat{h}$ from its spatial mean, following Wing and Emanuel (2014) and Wing and Cronin (2016), is given by 
$\frac{d \operatorname{var}(\hat{h})}{d t}=2\left\langle\widehat{h}^{\prime} F^{\prime}{ }_{K}\right\rangle+2\left\langle\widehat{h}^{\prime} N_{S}^{\prime}\right\rangle+2\left\langle\widehat{h^{\prime}} N_{L}^{\prime}\right\rangle-2\left\langle\widehat{h}^{\prime} \nabla_{h} \cdot \widehat{\mathbf{u} h}\right\rangle$,

where $F_{K}$ is the surface enthalpy flux, $N_{S}$ is the column shortwave flux convergence, $N_{L}$ is the column longwave flux convergence, and $-\nabla_{h} \cdot \widehat{\mathbf{u} h}$ is the horizontal convergence of the density-weighted column integral of the flux of frozen moist static energy. We refer to $-\widehat{h}^{\prime} \nabla_{h} \cdot \widehat{\mathbf{u} h}$ as the advective term, and we calculate it as a residual from the rest of the budget. A primed quantity denotes the spatial anomaly from the horizontal mean, which is indicated by angle brackets. We use a hat to indicate a density-weighted integral over the entire atmospheric column. Each of the terms on the right-hand side of Eq. (2) takes the form of a correlation between anomalies in $\hat{h}$ and anomalies in a source/sink of $\hat{h}$. A process that increases the $\hat{h}$ of an already high- $\hat{h}$ (i.e., moist) region therefore causes a positive tendency in the spatial variance, $\operatorname{var}(\hat{h})$, and is referred to as a "positive feedback" on aggregation and cyclogenesis.

Figure 5 shows the contributions to the growth rate of $\operatorname{var}(\hat{h})$. We find that feedbacks involving longwave radiation and surface fluxes dominate the initial development of a broad circulation, each contributing approximately equally, in both the rotating and nonrotating simulations (Figs. $5 \mathrm{c}, \mathrm{d}$ ). This indicates that the development of organized convection prior to genesis is similar to the initial phase of nonrotating selfaggregation (cf. days $25-40$ of the rotating simulation with days $0-15$ of the nonrotating simulation). The longwave-radiative feedbacks are composed primarily of cloud effects (Fig. 6); more clouds in the moist, high- $\hat{h}$ regions lead to reduced longwave cooling of the atmosphere, amplifying the $\hat{h}$ anomaly and acting as a positive feedback on aggregation.

As the tropical cyclone forms, intensifies, and is maintained (days $45-70$ of the rotating simulation), the surface flux feedback increases in magnitude beyond that of the radiative feedbacks, unlike in nonrotating aggregation where the surface flux feedback becomes negative as aggregation evolves (days $15-50$ of the nonrotating simulation). As described by Wing and Emanuel (2014), in nonrotating aggregation, reduced air-sea enthalpy disequilibrium in the moist, high- $\hat{h}$ regions eventually overwhelms the increased surface winds there, causing the surface flux feedback to become negative. This does not occur in the rotating simulation because of the much stronger winds that develop in association with the intensification of the tropical cyclone. However, we note that the longwave feedback is still significant during this time; its magnitude is between one-third and one-half of the surface flux feedback. The shortwave feedback is positive, but weak, throughout the simulation. Advection by the circulation damps anomalies in $\hat{h}$ throughout the rotating simulation, so that the growth in $\operatorname{var}(\hat{h})$ is entirely diabatically driven. This is in contrast to the nonrotating simulation, where advection by the circulation transports moist static energy from the dry to moist regions in the intermediate stages of aggregation (as in Bretherton et al. 2005; Muller and Held 2012; Wing and Emanuel 2014). These results are robust across the five-member ensemble of rotating RCE simulations, especially the finding that the surface flux and longwave radiation feedbacks contribute approximately equally in the period prior to genesis ( $\sim 15$ days prior to time 0 in Fig. $4 b$ ).

Tropical cyclogenesis is not completely prevented by eliminating radiative feedbacks, but it is delayed compared to when radiative feedbacks are enabled, occurring near the end of the rad_homog simulation (Figs. 2a,c). In two additional realizations of the rad_homog simulation (not shown), genesis occurs within the last 20 days of the simulation, but this is still outside the range of variability of the ensemble of full-physics simulations. For comparison, homogenizing the radiative heating rates completely prevents nonrotating aggregation (Muller and Held 2012; Wing 2014). In the LWrad_qfix simulation, tropical cyclogenesis occurs but is delayed slightly compared to the control simulation (Fig. 2d). This delay in genesis is not significant, as it is within the range of variability of the ensemble of full-physics simulations. In contrast, the same experiment prevents nonrotating aggregation (Wing 2014), suggesting that clear-sky longwave-water vapor feedbacks are more important for nonrotating than rotating aggregation. Both mechanism denial experiments suggest that rotating RCE aggregates more robustly than nonrotating RCE. Overall, the results of the feedback analysis and mechanism denial experiments indicate that while radiative feedbacks are not strictly necessary for cyclogenesis, they contribute significantly.

\section{Summary}

Spontaneous tropical cyclogenesis in a rotating radiative-convective equilibrium simulation is characterized by the spinup of a moist region, in contrast to self-aggregation in nonrotating simulations, which begins as a dry patch that is amplified and expands to force all the convection into a single region. We applied a variance budget for column-integrated frozen moist static energy to quantify the feedbacks leading to cyclogenesis. Our results agree qualitatively with the description of spontaneous genesis by Nolan et al. (2007) and Davis (2015) (who used different models than the 

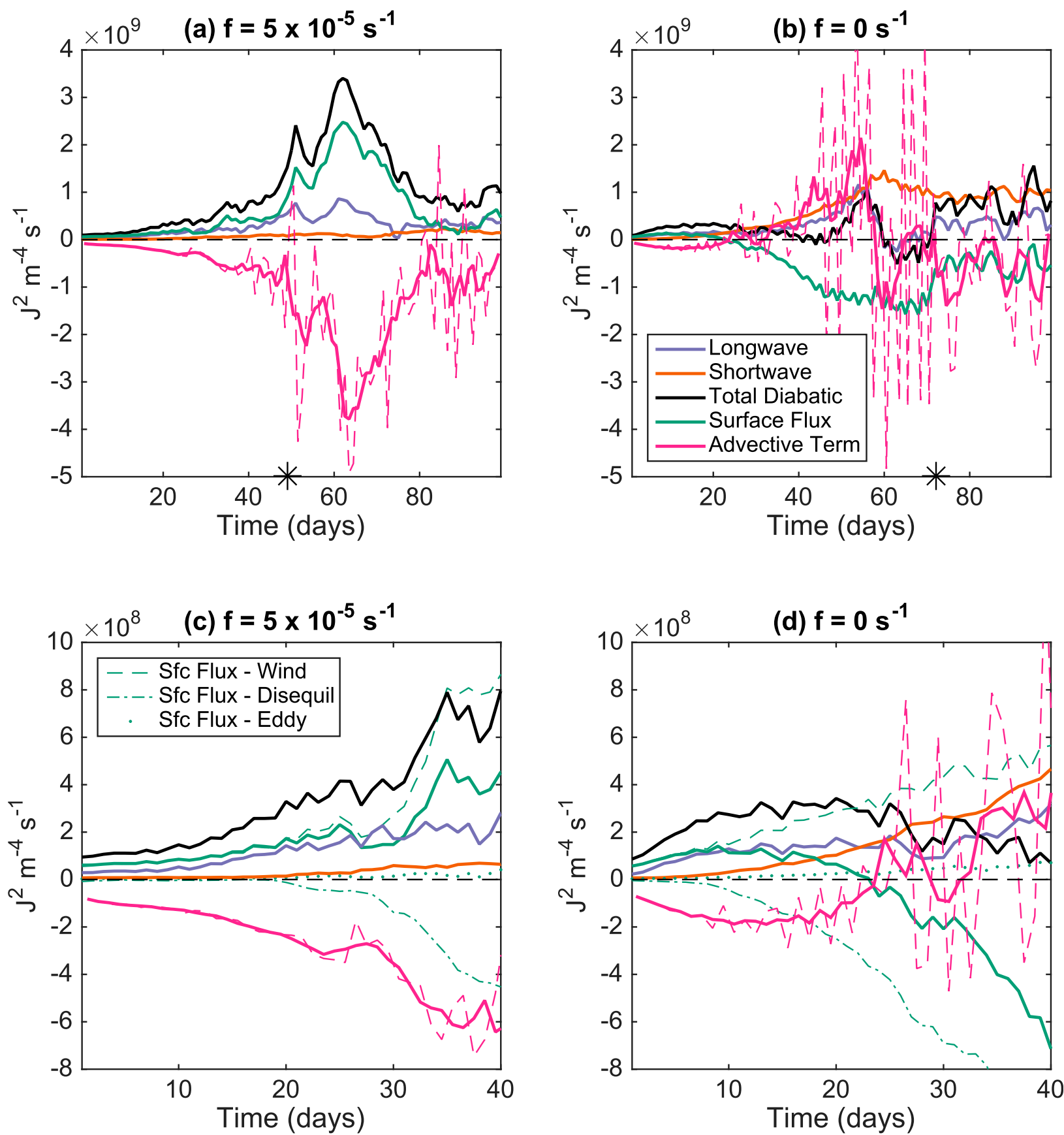

FIG. 5. Contributions to growth rate of $\operatorname{var}(\hat{h})$ for simulations at $305 \mathrm{~K}$ (a) with and (b) without rotation. Shown are the contributions from the column longwave flux convergence (blue), column shortwave flux convergence (red), surface enthalpy flux (green), advection by the circulation (pink dashed), and a 5-day running average of the advective contribution (pink). The black solid line is the sum of all the diabatic contributions and the zero line is shown with black dashes. The time to genesis in (a) and the time to a mature cluster in (b) are indicated by black asterisks on the $x$ axis. The first 40 days of the simulation are shown in more detail for the simulations (c) with and (d) without rotation [note the different axes compared to (a) and (b)]. The curves are as in (a) and (b) with the addition of the part of the surface flux feedback due to wind speed variations (green dashed), the part of the surface flux feedback due to air-sea enthalpy disequilibrium variations (green dotted-dashed), and the part of the surface flux feedback due to the correlation of wind speed and disequilibrium variations (green dotted). 


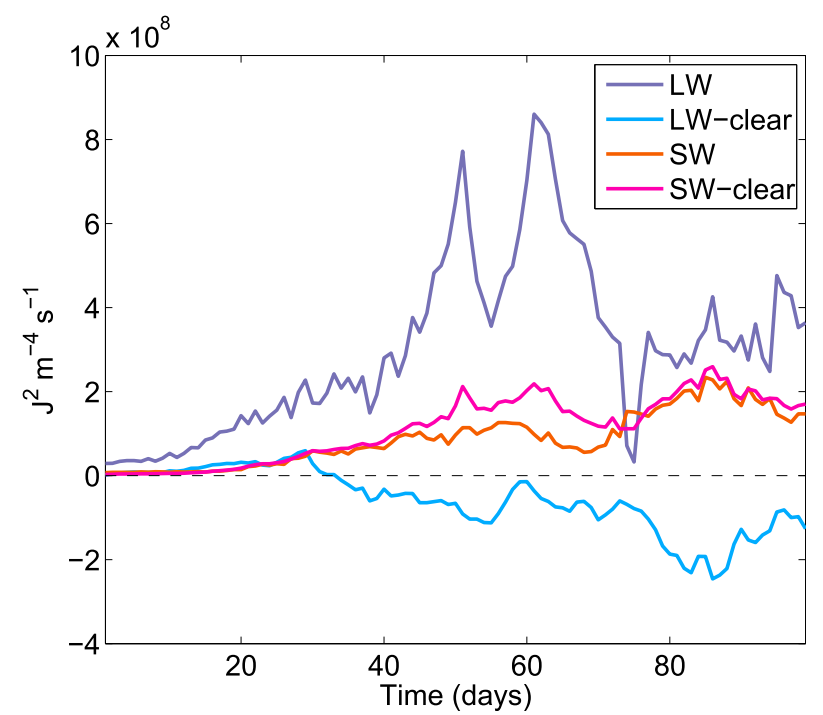

FIG. 6. Contributions to growth rate of $\operatorname{var}(\hat{h})$ for the simulation at $305 \mathrm{~K}$ with rotation $\left(f=5 \times 10^{-5} \mathrm{~s}^{-1}\right)$. Shown are the contributions from the total column longwave flux convergence (blue), clear-sky column longwave flux convergence (cyan), total column shortwave flux convergence (red), and clear-sky column shortwave flux convergence (pink). The zero line is shown with black dashes.

one used here) and explicitly show that the initial development of a broad circulation proceeds according to the same mechanisms that cause nonrotating selfaggregation: feedbacks involving longwave radiation and surface fluxes. As the tropical cyclone intensifies, the surface flux feedback dominates over the radiative feedbacks, unlike in nonrotating aggregation where the surface flux feedbacks become negative as aggregation evolves.

In nature, tropical cyclones are observed to form from preexisting disturbances rather than spontaneously, but this may simply be a faster or easier path to genesis than the highly idealized self-aggregation described here. Radiative-convective feedbacks may still play a role in amplifying those disturbances and aiding in localizing convection throughout the genesis process. Future work on this topic should include determining how these feedbacks operate when there are large-scale circulations, such as those associated with tropical waves.

Furthermore, even though the longwave-radiative feedback is not the dominant positive feedback in the later stages of genesis and intensification of the tropical cyclone, our analysis indicates that it is still a significant contributor to the variance budget (about one-half the magnitude of the surface flux feedback). This motivates further investigation into the role of radiation, in general, and especially longwave-cloud feedbacks, in the formation and evolution of tropical cyclones, a topic that has been underappreciated in the tropical cyclone literature.

Acknowledgments. We acknowledge high-performance computing support from Yellowstone (ark:/85065/ d7wd3xhc; Computational and Information Systems Laboratory 2012), provided by NCAR's Computational and Information Systems Laboratory, sponsored by the National Science Foundation. Allison A. Wing is supported by an NSF AGS Postdoctoral Research Fellowship under Award 1433251. We thank Shuguang Wang for helpful discussions and Marat Khairoutdinov for supplying the cloud-resolving model. We also thank Chris Davis for useful comments on the manuscript and helpful discussion, as well as an anonymous reviewer for their thoughtful feedback.

\section{REFERENCES}

Andersen, J. A., and Z. Kuang, 2012: Moist static energy budget of MJO-like disturbances in the atmosphere of a zonally symmetric aquaplanet. J. Climate, 25, 2782-2804, doi:10.1175/ JCLI-D-11-00168.1.

Arnold, N. P., and D. A. Randall, 2015: Global-scale convective aggregation: Implications for the Madden-Julian Oscillation. J. $A d v$. Model. Earth Syst., 7, 1499-1518, doi:10.1002/2015MS000498.

Boos, W. R., A. V. Fedorov, and L. Muir, 2016: Convective selfaggregation and tropical cyclogenesis under the hypohydrostatic rescaling. J. Atmos. Sci., 73, 525-544, doi:10.1175/ JAS-D-15-0049.1.

Bretherton, C. S., and M. F. Khairoutdinov, 2015: Convective selfaggregation feedbacks in near-global cloud-resolving simulations of an aquaplanet. J. Adv. Model. Earth Syst., 7, 17651787, doi:10.1002/2015MS000499.

_, P. N. Blossey, and M. Khairoutdinov, 2005: An energy-balance analysis of deep convective self-aggregation above uniform SST. J. Atmos. Sci., 62, 4273-4292, doi:10.1175/JAS3614.1.

$\mathrm{Bu}$, Y., R. Fovell, and K. Corbosiero, 2014: Influence of cloud radiative forcing on tropical cyclone structure. J. Atmos. Sci., 71, 1644-1662, doi:10.1175/JAS-D-13-0265.1.

Clough, S. A., M. W. Shephard, E. J. Mlawer, J. S. Delamere, M. J. Iacono, K. Cady-Pereira, S. Boukabara, and P. D. Brown, 2005: Atmospheric radiative transfer modeling: A summary of the AER codes. J. Quant. Spectrosc. Radiat. Transfer, 91, 233244, doi:10.1016/j.jqsrt.2004.05.058.

Computational Information Systems Laboratory, 2012: Yellowstone: IBM iDataPlex System (University Community Computing). National Center for Atmospheric Research, http:// n2t.net/ark:/85065/d7wd3xhc.

Davis, C. A., 2015: The formation of moist vortices and tropical cyclones in idealized simulations. J. Atmos. Sci., 72, 34993516, doi:10.1175/JAS-D-15-0027.1.

Dunkerton, T. J., M. T. Montgomery, and Z. Wang, 2009: Tropical cyclogenesis in a tropical wave critical layer: Easterly waves. Atmos. Chem. Phys., 9, 5587-5646, doi:10.5194/acp-9-5587-2009.

Hakim, G., 2011: The mean state of axisymmetric hurricanes in statistical equilibrium. J. Atmos. Sci., 68, 1364-1376, doi:10.1175/ 2010JAS3644.1. 
Held, I. M., and M. Zhao, 2008: Horizontally homogeneous rotating radiative-convective equilibria at GCM resolution. J. Atmos. Sci., 65, 2003-2013, doi:10.1175/2007JAS2604.1.

Iacono, M. J., J. S. Delamere, E. J. Mlawer, M. W. Shephard, S. A. Clough, and W. D. Collins, 2008: Radiative forcing by longlived greenhouse gases: Calculations with the AER radiative transfer models. J. Geophys. Res., 113, D13103, doi:10.1029/ 2008JD009944.

Jeevanjee, N., and D. M. Romps, 2013: Convective selfaggregation, cold pools, and domain size. Geophys. Res. Lett., 40, 994-998, doi:10.1002/grl.50204.

Khairoutdinov, M. F., and D. A. Randall, 2003: Cloud resolving modeling of the ARM summer 1997 IOP: Model formulation, results, uncertainties, and sensitivities. J. Atmos. Sci., 60, 607-625, doi:10.1175/1520-0469(2003)060<0607:CRMOTA >2.0.CO;2.

_ , and K. Emanuel, 2013: Rotating radiative-convective equilibrium simulated by a cloud-resolving model. J. Adv. Model. Earth Syst., 5, 816-825, doi:10.1002/2013MS000253.

Melhauser, C., and F. Zhang, 2014: Diurnal radiation cycle impact on the pregenesis environment of Hurricane Karl (2010). J. Atmos. Sci., 71, 1241-1259, doi:10.1175/JAS-D-13-0116.1.

Mlawer, E. J., S. J. Taubman, P. D. Brown, M. J. Iacono, and S. A. Clough, 1997: Radiative transfer for inhomogeneous atmospheres: RRTM, a validated correlated-k model for the longwave. J. Geophys. Res., 102, 16 663-16 682, doi:10.1029/97JD00237.

Muller, C. J., and I. M. Held, 2012: Detailed investigation of the self-aggregation of convection in cloud-resolving simulations. J. Atmos. Sci., 69, 2551-2565, doi:10.1175/JAS-D-11-0257.1.

- and S. Bony, 2015: What favors convective aggregation and why? Geophys. Res. Lett., 42, 5626-5643, doi:10.1002/2015GL064260.

Nolan, D. S., E. D. Rappin, and K. A. Emanuel, 2007: Tropical cyclonegenesis sensitivity to environmental parameters in radiative-convective equilibrium. Quart. J. Roy. Meteor. Soc., 133, 2085-2107, doi:10.1002/qj.170.

Raymond, D. J., S. L. Sessions, and Ž. Fuchs, 2007: A theory for the spinup of tropical depressions. Quart. J. Roy. Meteor. Soc., 133, 1743-1754, doi:10.1002/qj.125.

Reed, K. A., and D. R. Chavas, 2015: Uniformly rotating global radiative-convective equilibrium in the Community Atmosphere Model, version 5. J. Adv. Model. Earth Syst., 7, 19381955, doi:10.1002/2015MS000519.

Schecter, D. A., 2011: Evaluation of a reduced model for investigating hurricane formation from turbulence. Quart. J. Roy. Meteor. Soc., 137, 155-178, doi:10.1002/qj.729.
_ 2013: Relationships between convective asymmetry, imbalance and intensity in numerically simulated tropical cyclones. Tellus, 65A, 20168, doi:10.3402/tellusa.v65i0.20168.

Sessions, S. L., S. Sugaya, D. J. Raymond, and A. H. Sobel, 2010: Multiple equilibria in a cloud-resolving model using the weak temperature gradient approximation. J. Geophys. Res., 115, D12110, doi:10.1029/2009JD013376.

Sobel, A., and E. Maloney, 2012: An idealized semi-empirical framework for modeling the Madden-Julian oscillation. J. Atmos. Sci., 69, 1691-1705, doi:10.1175/JAS-D-11-0118.1.

— , G. Bellon, and J. Bacmeister, 2007: Multiple equilibria in a single-column model of the tropical atmosphere. Geophys. Res. Lett., 34, L22804, doi:10.1029/2007GL031320.

- S. Wang, and D. Kim, 2014: Moist static energy budget of the MJO during DYNAMO. J. Atmos. Sci., 71, 4276-4291, doi:10.1175/JAS-D-14-0052.1.

Stephens, G. L., S. van den Heever, and L. Pakula, 2008: Radiativeconvective feedbacks in idealized states of radiative-convective equilibrium. J. Atmos. Sci., 65, 3899-3916, doi:10.1175/ 2008JAS2524.1.

Tompkins, A. M., 2001: Organization of tropical convection in low vertical wind shears: The role of water vapor. J. Atmos. Sci. 58, 529 545, doi:10.1175/1520-0469(2001)058<0529:OOTCIL >2.0.CO;2.

Wing, A. A., 2014: Physical mechanisms controlling selfaggregation of convection in idealized numerical modeling simulations. Ph.D. dissertation, Massachusetts Institute of Technology, 146 pp. [Available online at http://dspace.mit. edu/handle/1721.1/90606.]

_ , and K. A. Emanuel, 2014: Physical mechanisms controlling self-aggregation of convection in idealized numerical modeling simulations. J. Adv. Model. Earth Syst., 6, 59-74, doi:10.1002/2013MS000269.

— long channel geometry. Quart. J. Roy. Meteor. Soc., 142, 1-15, doi:10.1002/qj.2628.

Yokoi, S., and A. H. Sobel, 2015: Seasonal march and intraseasonal variability of the moist static energy budget over the eastern Maritime Continent during CINDY2001/ DYNAMO. J. Meteor. Soc. Japan, 93A, 81-100, doi:10.2151/jmsj.2015-041.

Zhou, W., I. M. Held, and S. T. Garner, 2014: Parameter study of tropical cyclones in rotating radiative-convective equilibrium with column physics and resolution of a 25-km GCM. J. Atmos. Sci., 71, 1058 1068, doi:10.1175/JAS-D-13-0190.1. 